\title{
Reacciones adversas a medicamentos en un hospital de tercer nivel en Ciudad Victoria, México
}

\section{Adverse drug reactions in a tertiary hospital at Ciudad Victoria, Mexico}

\author{
José Sánchez-Guevara ${ }^{1 *}$, Patricia Rivera-Vázquez ${ }^{2}$ y Elizabeth Llanas-Olmos ${ }^{3}$ \\ ${ }^{1}$ Departamento de la Subdirección de Atención Hospitalaria; ${ }^{2}$ Departamento de la Coordinación de Investigación en Enfermería; ${ }^{3}$ Departamento de la Gestoría de \\ Calidad. Hospital Regional de Alta Especialidad Bicentenario 2010, Ciudad Victoria, Tamaulipas. México
}

\begin{abstract}
The objective of the study was to identify the adverse drug reactions (ADR) in a hospital of third level of care in Ciudad Victoria, Tamps., through a descriptive-cross-sectional study reviewing the clinical charts of patients who presented adverse reactions to the drugs during the years 2014-2018. Results show that adverse events were reported in 30 patients, mostly female (60\%), young adults (37\%), with acute pathology (53\%), concomitant disease (33\%), without a history of ADR (93\%), who received antibiotics (30\%), analgesics (23\%) and others (47\%). The patients presented cutaneous reaction (47\%), tachycardia (17\%), dyspnea (13\%) and other less frequent. All patients were followed up. We conclude that pharmacovigilance facilitates the identification of ADR for a safe and quality care of patients.
\end{abstract}

Key words: Adverse reaction. Medications. Pharmacovigilance.

\section{Resumen}

El objetivo del estudio fue identificar las reacciones adversas a medicamentos (RAM) en un hospital de tercer nivel de atención en Ciudad Victoria, Tamps., mediante un estudio descriptivo transversal con expedientes de pacientes que presentaron RAM durante los años 2014-2018. Los resultados muestran que se reportaron eventos adversos en 30 pacientes, en su mayoría del sexo femenino (60\%), adultos jóvenes (37\%), con patología aguda (53\%), enfermedad concomitante (33\%) y sin antecedente de RAM (93\%). Los pacientes que presentaron RAM habían recibido antibióticos (30\%), analgésicos (23\%) y otros (47\%). Las principales manifestaciones fueron reacción cutánea (47\%), taquicardia (17\%), disnea (13\%) y otras menos frecuentes. A todos los pacientes se les dio seguimiento. Se concluye que la farmacovigilancia facilita la identificación de RAM para una atención segura y de calidad a los pacientes.

Palabras clave: Reacción adversa. Medicamento. Farmacovigilancia.

\section{Correspondence:}

*José Sánchez-Guevara

E-mail: jose.sanchez@hraev.gob.mx
Date of reception: 09-07-2018

Date of acceptance: $30-01-2020$

DOI: 10.24875/HMCM.20000142
Available online: 02-04-2020 Hosp Med Clin Manag. 2020;13:20-5

2604-0018 / @ 2020 Mexican Regional Hospitals of High Specialty and Federal Hospitals. Published by Permanyer. This is an open access article under the CC BY-NC-ND license (http://creativecommons.org/licenses/by-nc-nd/4.0/). 


\section{INTRODUCCIÓN}

La Organización Mundial de la Salud define como reacción adversa a un medicamento (RAM) a «cualquier respuesta a un fármaco que es nociva, no intencionada y que se produce a dosis habituales para la profilaxis, diagnóstico o tratamiento". Son efectos no deseados ni intencionados de un medicamento, incluidos los efectos idiosincráticos, que se producen durante su uso adecuado, de tal forma que los pacientes suelen responder de manera diferente a una determinada pauta de tratamiento, por lo que hay que considerar que, además de las propiedades farmacéuticas de los medicamentos, existen algunas características del paciente que predisponen a los RAM ${ }^{1}$.

Al respecto, la farmacovigilancia es una más de las actividades destinadas a la detección, identificación, cuantificación, evaluación y prevención de los posibles riesgos derivados del uso de los medicamentos y vacunas en seres humanos. Es una actividad de responsabilidad compartida entre todos los agentes relacionados a los medicamentos que se utilicen en el tratamiento de seres humanos, como lo es el equipo multidisciplinario en las instituciones de salud².

En el mundo se estima que existe una incidencia importante de reacciones adversas que pueden ser fatales para los pacientes. Estas reacciones están entre las principales causas de muerte, lo que representa un importante problema clínico para el personal de salud, ya que la mayoría de estas se reportan como graves y probablemente evitables ${ }^{3}$. Diversos estudios revelan una incidencia de sospechas de RAM en pacientes hospitalizados del $13 \%{ }^{4}$.

Entre los factores de riesgo reportados está la edad, especialmente en los extremos de la vida, donde se es más susceptible a las RAM, ya que durante la edad avanzada se utilizan con frecuencia hipnóticos, diuréticos, antiinflamatorios no esteroideos, antihipertensivos, psicotrópicos (entre otros) y, a pesar de que los niños, sobre todo los recién nacidos, difieren de los adultos, existen fármacos que se asocian con mayor riesgo a las RAM, como los antiarrítmicos y los antibióticos, entre otros.

El sexo de mayor riesgo es el femenino. Otro riesgo es la polifarmacia, debido a que se pueden producir interacciones entre fármacos que compiten por el mismo receptor o que actúan sobre el mismo sistema fisiológico.
La interacción puede aumentar o disminuir los efectos de los fármacos implicados y puede causar toxicidad inesperada, provocando una reacción adversa. Otros factores se relacionan con la comorbilidad, ya que si además de la enfermedad que se está tratando el paciente sufre otra enfermedad (como una alteración renal, hepática o cardiaca), se pueden requerir precauciones especiales para el tratamiento de las enfermedades intercurrentes y los fármacos concurrentes interactivos, especialmente cuando existen antecedentes de RAM en el paciente ${ }^{4,5}$.

La tasa más alta de sospecha de reacciones adversas se reporta como probable y grave especialmente en los pacientes hospitalizados con estancia prolongada, pacientes del sexo femenino y pacientes con más de cuatro fármacos. Respecto a la edad, la mayoría de autores difiere, ya que en algunos estudios existe mayor presencia de RAM en el grupo de edad de 21-30 años ${ }^{5}$, mientras que en otros es la población de edad avanzada la más afectada ${ }^{6}$.

Las reacciones adversas más frecuentes ocurridas durante la estancia hospitalaria son cutáneas, generales, vasculares y cardiacas ${ }^{4-6}$. Algunos estudios muestran que también se presentan reacciones digestivas y neurológicas relacionadas a la medicación ${ }^{7-9}$. En cuanto a las clases de fármacos reportados como de riesgo para RAM se reportan los antibacterianos, antitrombóticos inhibidores de los CVA, antiinflamatorios no esteroideos y diuréticos ${ }^{10}$. Los activos más comúnmente reportados en las sustancias asociadas con un evento adverso son el tramadol, la dipirona, la glibenclamida y la furosemida ${ }^{11}$, además de los corticoesteroides tópicos, el fosfato sódico de betametasona y el clobetasol, que inducen el máximo número de reacciones ${ }^{12}$.

Algunos autores señalan que las RAM se reportan en su mayoría como posibles y leves, siendo la mayor parte prevenibles ${ }^{13-15}$. Por ello, es de gran relevancia para las instituciones de salud mantener de manera activa protocolos de fármaco vigilancia con la finalidad de identificar factores de riesgo que puedan condicionar la presencia de reacciones adversas que pongan en peligro no solo la salud ya comprometida en el paciente, sino también su vida.

Por lo anterior, el presente estudio tuvo como objetivo identificar las reacciones adversas a medicamentos en un hospital de tercer nivel de atención en Ciudad Victoria, Tamps. 


\section{$18(40 \%)$}

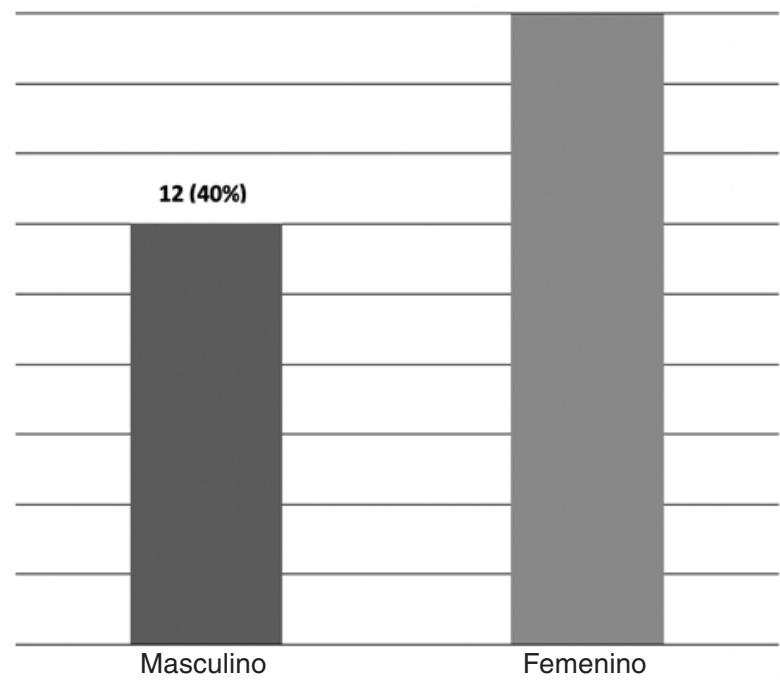

Figura 1. Distribución de los pacientes que presentaron reacciones adversas a medicamentos según su sexo $(n=30)$.

\section{MÉTODOS}

Investigación clínica, no experimental, realizada por medio de un estudio retrospectivo, descriptivo, transversal y observacional. Se seleccionó la muestra mediante muestreo no probabilístico por disponibilidad, y estuvo conformada por 30 pacientes que cumplieron con los siguientes criterios de inclusión: ser paciente hospitalizado en cualquiera de las áreas de servicio del hospital, de ambos sexos, de edad indistinta, que presentaron alguna RAM durante su estancia hospitalaria en el periodo comprendido entre julio de 2014 y junio de 2018. Se excluyeron pacientes con antecedentes de alergia registrados en la historia clínica.

Los datos fueron registrados en formato diseñado ex profeso por los autores para fines de la investigación considerando datos sociodemográficos como edad, sexo, diagnóstico médico, tipo de medicamento utilizado, reacción adversa identificada y seguimiento del paciente. Los datos fueron capturados y analizados mediante el programa Statistical Package for the Social Sciences ${ }^{\circledR}$ (SPSS) v.21 para Windows mediante estadística descriptiva.

La presente investigación estuvo apegada a lo dispuesto en la Declaración de Helsinki (2013) y a las consideraciones éticas y legales enmarcadas en la Ley General

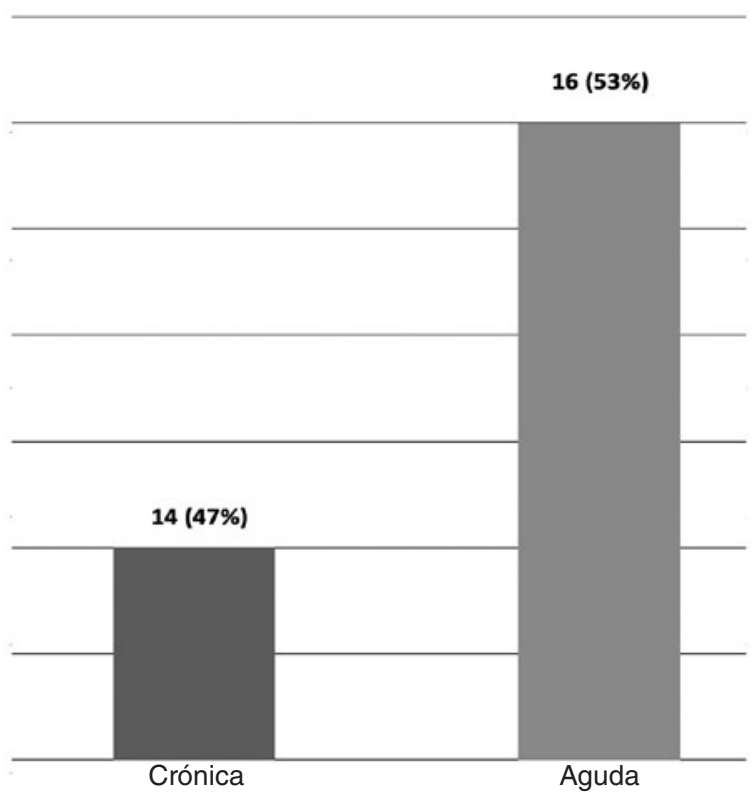

Figura 2. Tipo de patología de los pacientes que presentaron reacciones adversas a medicamentos $(n=30)$.

de Salud en Materia de Investigación en Salud vigente en México, por lo que es considerada como investigación sin riesgo.

\section{RESULTADOS}

Al término del estudio encontramos que se reportaron un total de 32 RAM en 30 pacientes hospitalizados, que se presentaron en el periodo comprendido entre 2014 y 2018 de la siguiente manera: 1 (3.3\%) en el año 2014 , 5 (16.7\%) en 2015, 5 (16.7\%) en 2016, 6 (20\%) en 2017 y los $13(43 \%)$ restantes durante el año 2018.

La media de edad de los pacientes que presentaron RAM fue 44 años (mínimo de 12 años y máximo de 82 , en un rango de 70 años); 18 (60\%) pacientes fueron del sexo femenino y 12 (40\%) del masculino (Fig. 1).

Respecto al tipo de patología por la cual fueron hospitalizados los pacientes encontramos que 16 (53\%) pacientes cursaban con patología aguda y 14 (47\%) patología crónica (Fig. 2). Las patologías que presentaron RAM fueron en su mayoría de tipo oncológico, ello observado en 7 (23\%) pacientes (leucemia linfoblástica aguda 2, adenocarcinoma de colon 3 , cáncer de recto 1 y cáncer cervicouterino 1); el resto presentó otro tipo de patologías de diversa etiología, 23 (77\%) especialmente de tipo 


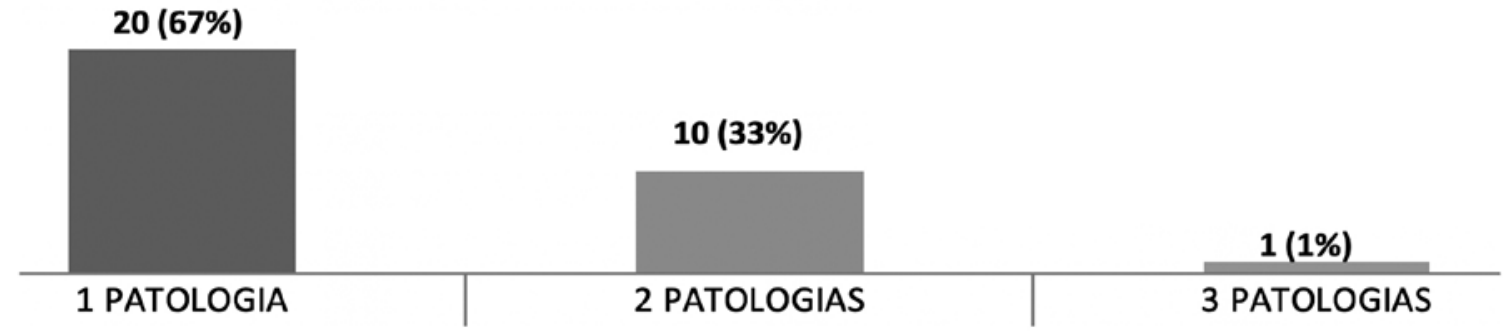

Figura 3. Patologías concomitantes en los pacientes que presentaron reacciones adversas a medicamentos $(n=30)$.

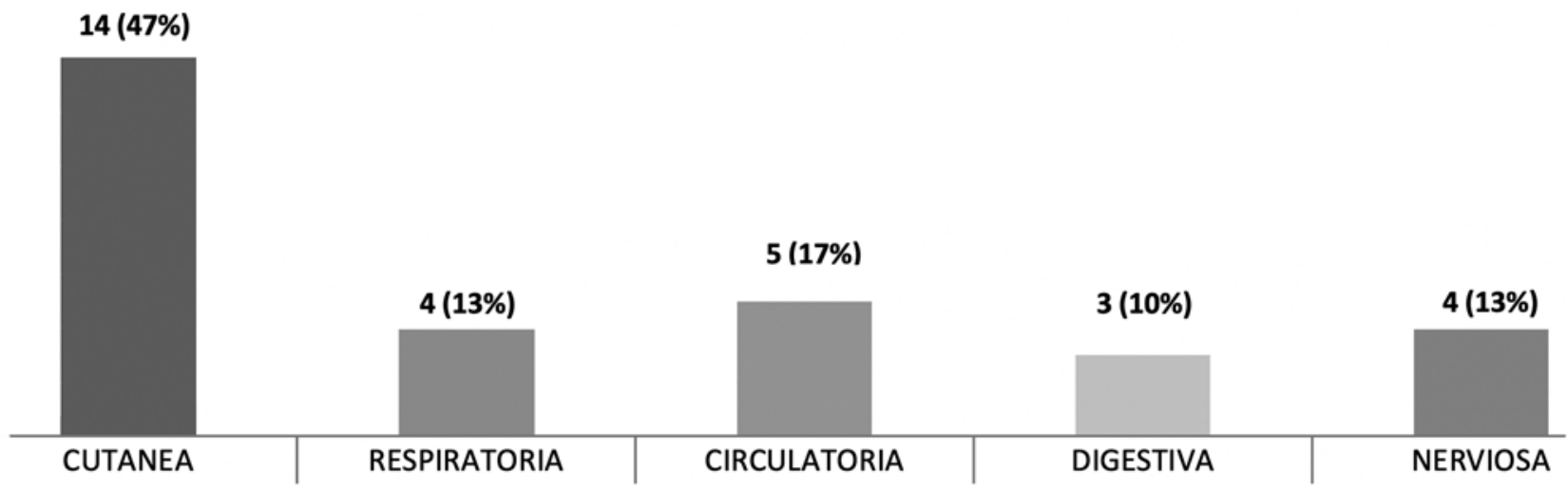

Figura 4. Tipos de medicamentos relacionados con la presencia de reacciones adversas a medicamentos $(n=30)$.

infeccioso. Solamente 10 (33\%) de los pacientes cursaba con enfermedad concomitante; de ellos, solo $1(1 \%)$ cursaba con 3 enfermedades crónicas (hipertensión arterial, obesidad, diabetes tipo 2); el resto, 20 (67\%), cursaba con solo una patología (Fig. 3).

Solamente en 2 (7\%) de los pacientes se encontró antecedente de RAM en su historia clínica. Respecto al tipo de medicamento, encontramos que las RAM fueron posteriores a la administración de antibióticos en 9 (30\%), analgésicos en 7 (23\%), gastrocinéticos en 3 (10\%), anticancerosos en $7(23 \%)$ y otros en $4(13 \%)$ (Fig. 4).

De los pacientes que presentaron RAM, 14 (47\%) presentó reacción cutánea (rash, dermatitis); 4 (13\%) reacción respiratoria, especialmente disnea; 5 (17\%) presentaron taquicardia, hipotensión; 3 (10\%) náuseas, mareo y diarrea; y 4 (13\%) pacientes presentaron ansiedad, agitación, cefalea o somnolencia, entre otras reacciones nerviosas (Fig. 5).

Es importante mencionar que a los 30 (100\%) pacientes se les dio seguimiento y tratamiento médico, vigilando su evolución. Las RAM se consideraron como leves y probables.

\section{DISCUSIÓN}

Las reacciones adversas que se identificaron en los pacientes hospitalizados en el Hospital Regional de Alta Especialidad de Ciudad Victoria, Tamaulipas, durante el periodo 2014-2018 fueron en su mayoría de tipo cutáneo (rash, dermatitis), como se menciona en la literatura, seguido de reacciones respiratorias y circulatorias (disnea, taquicardia, hipotensión) 4-6 $^{4}$. A diferencia con otros reportes, los pacientes estudiados presentaron reacciones digestivas (náuseas, mareo y diarrea) y reacciones relacionadas con el sistema nervioso (cefalea o somnolencia, entre otras) ${ }^{7-9}$.

Los pacientes que presentaron RAM en el hospital fueron en su mayoría adultos jóvenes, a diferencia de lo encontrado por otros autores, quienes mencionan que las RAM se observan con mayor frecuencia en los extremos de la vida, como son los neonatos y los adultos mayores ${ }^{4,6,14,15}$. En relación con el sexo, en el hospital 


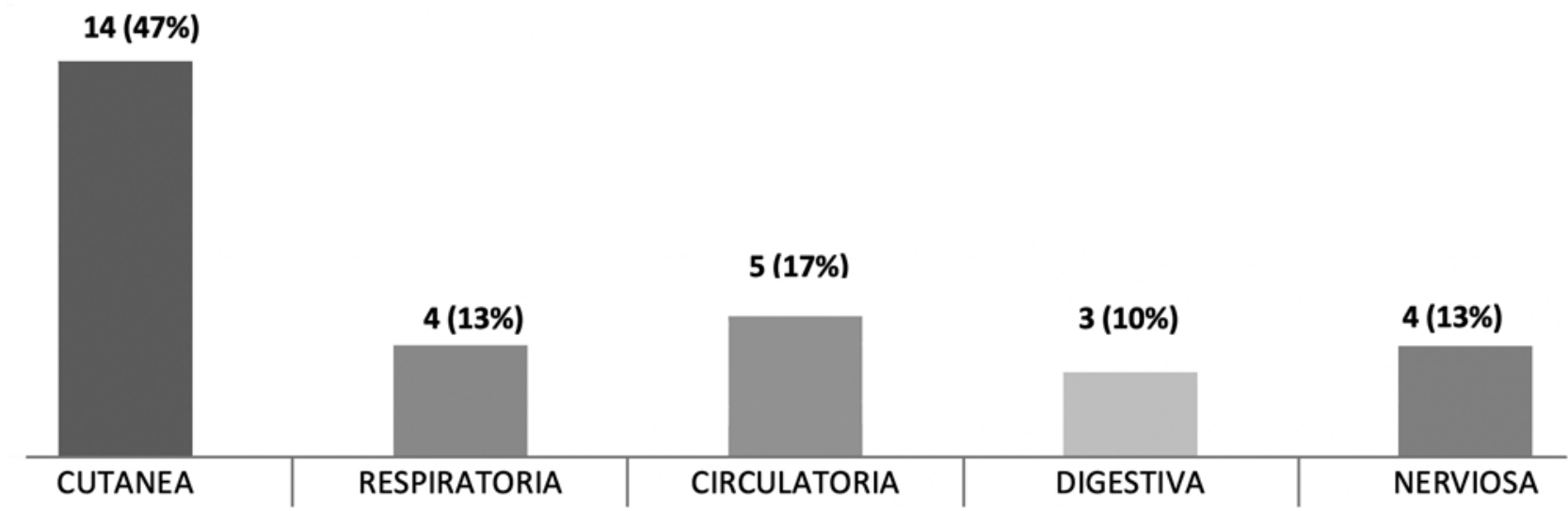

Figura 5. Tipo de reacciones adversas a medicamentos que presentaron los pacientes hospitalizados $(n=30)$.

las RAM se presentaron con mayor frecuencia en las mujeres ${ }^{4,5,11}$.

Respecto al tipo de patología, más de la mitad de los pacientes cursaban con patología aguda, a diferencia de otros estudios que mencionan que un factor de riesgo para RAM en los pacientes es cursar con patología crónica ${ }^{4-6,14}$. De tal forma, en el estudio solamente se encontraron dos pacientes con historia de RAM, contrario a lo descrito en la literatura, en donde se comenta que la mayoría de los pacientes con RAM son aquellos con historia previa de estas y por lo tanto son prevenibles $3,5,14$.

Los medicamentos relacionados con la presencia de RAM en los pacientes fueron en su mayoría antibióticos, analgésicos y anticancerosos ${ }^{4}$ (vancomicina, ceftriaxona, rifaximina, capecitabina, ciprofloxacino), entre otros menos frecuentes como la metoclopramida, butilhioscina, etc. Esto es contrario a lo mencionado en otros estudios $^{10}$, que describen como posibles causantes de RAM a medicamentos como antitrombóticos, antiinflamatorios no esteroideos y diuréticos, como el tramadol, la dipirona, la glibenclamida, los corticoesteroides y la furosemida, entre otros. A todos los pacientes estudiados se les dio seguimiento, considerando que las RAM presentadas se clasificaron como posibles y leves, de forma similar a otros estudios ${ }^{13-15}$.

\section{CONCLUSIÓN}

La farmacovigilancia que se desarrolla en el hospital facilita la identificación de reacciones adversas a medicamentos comúnmente utilizados en los pacientes. Ello permite establecer estrategias de mejora continua, ya que la mayoría de las RAM se considera como prevenible, por lo que es imperativo integrar a todo el equipo de salud para una atención segura y de calidad a los pacientes.

\section{AGRADECIMIENTOS}

A las autoridades del Hospital Regional De Alta Especialidad de Ciudad Victoria, Tamps., y al personal de epidemiología por su valiosa aportación para el desarrollo del presente estudio.

\section{FINANCIAMIENTO}

La presente investigación no ha recibido ninguna beca específica de agencias de los sectores público, comercial, o sin ánimo de lucro.

\section{CONFLICTO DE INTERESES}

Ninguno.

\section{RESPONSABILIDADES ÉTICAS}

Protección de personas y animales. Los autores declaran que para esta investigación no se han realizado experimentos en seres humanos ni en animales.

Confidencialidad de los datos. Los autores declaran que han seguido los protocolos de su centro de trabajo sobre la publicación de datos de pacientes. 
Derecho a la privacidad y consentimiento informado. Los autores declaran que en este artículo no aparecen datos de pacientes.

\section{BIBLIOGRAFÍA}

1. Medicamentos esenciales y productos de salud. Un recurso de la Organización Mundial de la Salud. Formulario Modelo de la OMS 2004 [Internet]. Organización Mundial de la Salud; 2004. Disponible en: http://who.int/ medicinedocs/es/d/Js5422s/4.4.html

2. Norma Oficial Mexicana NOM 220-SSA1-2016, Instalación y operación de la farmacovigilancia.

3. Lazarou J, Pomeranz BH, Corey PN. Incidence of adverse drug reactions in hospitalized patients. Meta-analysis of prospective studies. JAMA. 1998;279(15):1200-5.

4. Ratan J, Mangala L, Sukirti D, Debeeka H, Muralikotni M. A study on adverse drug reactions in a tertiary care hospital of North India. Alexandria J Med. 2017;53(2):151-6.

5. Mudigubba M, Rajarshekarachari Y, Dahiya S. Evaluation of predisposing factor associated with suspected adverse drug reactions of hospitalized patients. J Young Pharm. 2018;10(2):202-7.

6. Giardina C, Cutroneo PM, Mocciaro E, Russo GT, Mandraffino G, Basile G. Adverse drug reactions in hospitalized patients. Results of the FORWARD (Facilitation of Reporting in Hospital Ward) Study. Front Pharmacol. 2018;9(1):350.
7. RozenfeldI S, Giordanil F, Coelholl S. Eventos adversos a medicamentos en un hospital terciario: estudio piloto con rastreadores. Rev Saúde Públ. 2013;47(6):1102-11.

8. Novoa-Heckel G, Asbún-Bojalil J, Sevilla-González MDL. Responsabilidad profesional aplicada a la farmacovigilancia: un estudio de caso en México. Acta Bioética. 2016;22(2):269-80

9. Casas-Gross I, Gross-Fernández C, Ramos-Hernández L, Roldán Malo R. Reacciones adversas a medicamentos en el Hospital Provincial Docente Clínico-quirúrgico "Saturnino Lora Torres." MEDISAN. 2016;20(8):1077.

10. Mendo Alcolea N, Cala Calviño L, Leyva Miranda T, Álvarez Escalante L. Reacciones adversas medicamentosas en pacientes con cáncer atendidos en el Hospital Oncológico Docente "Conrado Benítez". MEDISAN. 2017;21(11):3145.

11. Sánchez I, Amador C, Cristian P, Correa G, Amador R. Impacto clínico de un sistema de fármaco-vigilancia activa realizado por un farmacéutico en el reporte y subnotificación de reacciones adversas a medicamentos. Rev Med Chile. 2014;142:998-1005

12. González- Guibert L, Puga Torres M, Lara Fernández H, Miranda Gómez 0 , Labadiño Cantillo A. Reacciones adversas a medicamentos en una unidad quirúrgica de urgencias. Rev Cub Med Militar. 2014;43(2):216-27.

13. Botero Aguirre JP, Restrepo Hamid AM. Caracterización de las reacciones adversas a medicamentos reportadas al programa de Farmacovigilancia de un hospital de alta complejidad y factores asociados con su evitabilidad. Rev Medicina UPB. 2013;32(2):129-37.

14. Jiménez López G, García Fariñas A, Gálvez González AM, Orta IA, Lara Bastanzuri MC, Calvo Barbado DM. Medicamentos notificados como productores de reacciones adversas graves en Cuba en un periodo de 10 años. Rev Cub Salud Públ. 2014;40(4):263-75.

15. Chaves M. Caracterización de reacciones adversas a medicamentos en adultos mayores de 44 años en Bogotá, D.C. Rev Biomed. 2015;35(1):34-42. 